\title{
Dietary inflammatory index and type 2 diabetes risk in a prospective cohort of 70,991 women followed for 20 years: the mediating role of BMI
}

\author{
Nasser Laouali ${ }^{1,2}$. Francesca Romana Mancini ${ }^{1,2} \cdot$ Mariem Hajji-Louati $^{1,2} \cdot$ Douae El Fatouhi $^{1,2} \cdot$ Beverley Balkau $^{1,2}$. \\ Marie-Christine Boutron-Ruault ${ }^{1,2}$ • Fabrice Bonnet ${ }^{3}$ • Guy Fagherazzi ${ }^{1,2,4}$
}

Received: 11 March 2019 / Accepted: 26 June 2019 / Published online: 9 August 2019

(C) Springer-Verlag GmbH Germany, part of Springer Nature 2019

\begin{abstract}
Aims/hypothesis Diet is one of the main lifestyle-related factors that can modulate the inflammatory process. Surprisingly the dietary inflammatory index (DII) has been little investigated in relation to type 2 diabetes, and the role of BMI in this relationship is not well established. We studied this association and the role of BMI in the inflammatory process in a large population-based observational study.

Methods A total of 70,991 women from the E3N (Etude Epidémiologique auprès de femmes de la Mutuelle Générale de l'Education Nationale) cohort study were followed for 20 years. Incident type 2 diabetes cases were identified using diabetesspecific questionnaires and drug reimbursement insurance databases, and 3292 incident cases were validated. The DII was derived from a validated food frequency questionnaire. Multivariable Cox regression models estimated HRs and 95\% CIs between DII and incident type 2 diabetes. Interactions were tested between DII and BMI on incident type 2 diabetes and a mediation analysis of BMI was performed.

Results Higher DII scores, corresponding to a higher anti-inflammatory potential of the diet, were associated with a lower risk of type 2 diabetes. Compared with the 1 st quintile group, women from the 2 nd quintile group (HR 0.85 [95\% CI 0.77, 0.94]) up to the 5th quintile group (HR 0.77 [95\% CI 0.69, 0.85]) had a lower risk of type 2 diabetes before adjustment for BMI. There was an interaction between DII and BMI on type 2 diabetes risk ( $\left.p_{\text {Interaction }}<0.0001\right)$. The overall association was partly mediated by BMI $(58 \%)$.

Conclusions/interpretation Our findings suggest that a higher anti-inflammatory potential of the diet is associated with a lower risk of type 2 diabetes, and the association may be mediated by BMI. These results may improve our understanding of the mechanisms underlying the role of diet-related anti-inflammation in the pathogenesis of type 2 diabetes in women. Further studies are warranted to validate our results and evaluate whether the results are similar in men.
\end{abstract}

Keywords BMI, Body mass index $\cdot$ Cohort $\cdot$ Diet inflammation $\cdot$ Mediation analysis $\cdot$ Prevention $\cdot$ Risk $\cdot$ Type 2 diabetes

Nasser Laouali and Francesca Romana Mancini are joint first authors.

Electronic supplementary material The online version of this article (https://doi.org/10.1007/s00125-019-04972-0) contains peer-reviewed but unedited supplementary material, which is available to authorised users.

Guy Fagherazzi

guy.fagherazzi@gustaveroussy.fr

1 Centre for Research in Epidemiology and Population Health (CESP), Inserm (Institut National de la Santé et de la Recherche Médicale) U1018, Generations and Health Across Generations, Gustave Roussy Institute, 114 rue Edouard Vaillant, 94805 Villejuif Cedex, France
2 Faculte de Medecine, UPS-UVSQ-Paris-Saclay University, Le Kremlin-Bicêtre, France

3 Service d'Endocrinologie, Groupe Hospitalier Paris St-Joseph, Paris, France

4 Department of Population Health, Luxembourg Institute of Health (LIH), Strassen, Luxembourg 


\section{Research in context}

\section{What is already known about this subject?}

- Evidence suggests that inflammation plays a key role in the pathogenesis of type 2 diabetes

- Diet is one of the main lifestyle-related factors that can modulate the inflammatory process

- Few epidemiological studies have evaluated the link between dietary inflammatory indexes (DIIs) and type 2 diabetes; results are conflicting and the role of BMI is not well established

\section{What is the key question?}

- Is the dietary inflammatory index, developed to assess the overall inflammatory potential of the diet, associated with type 2 diabetes incidence, and is the association mediated or modified by BMI?

\section{What are the new findings?}

- In our study, an elevated anti-inflammatory potential of the diet was associated with a lower risk of type 2 diabetes

- The overall association between DII and type 2 diabetes risk was partly mediated by BMI

How might this impact on clinical practice in the foreseeable future?

- These results may improve our understanding of the mechanisms underlying the role of diet-related inflammation in the pathogenesis of type 2 diabetes and help to design more efficient nutritional preventative interventions

\author{
Abbreviations \\ ADII Adapted dietary inflammatory index \\ CDE Controlled direct effect \\ DII Dietary inflammatory index \\ E3N Etude Epidémiologique auprès de femmes de la \\ Mutuelle Générale de l'Education Nationale \\ NDE Natural direct effect \\ NIE Natural indirect effect
}

\section{Introduction}

The global prevalence of type 2 diabetes is increasing, from 415 million adults in 2015 [1] rising to 629 million people expected to develop diabetes by 2045 [2]. While many metabolic and lifestyle factors are known to contribute to the development of type 2 diabetes and its complications, the involvement of the immune system in the pathogenesis of type 2 diabetes has recently been gaining interest. A considerable body of evidence suggests that inflammation plays a key role in the pathogenesis of type 2 diabetes [3, 4]. Obesity, hyperglycaemia, insulin resistance, and the overexpression of proinflammatory proteins such as C-reactive protein and cytokines (IL- $1 \beta$, IL- 6 and TNF- $\alpha$ ) induce chronic inflammation in type 2 diabetes [5]. In addition, environmental, behavioural and psychosocial factors can stimulate inflammation in times of stress [6]. Among the modifiable factors, diet is one of the main lifestyle-related factors which can modulate the inflammatory process [7]. Several foods and food components have an impact on blood concentrations of inflammatory markers, including cytokines, chemokines, acute-phase proteins, soluble adhesion molecules and cytokine receptors $[8,9]$.

Recently, dietary inflammatory indexes (DII) have been developed based on evidence linking diet with inflammation [10-12]. Only four cross-sectional studies and one prospective study have evaluated the link between these indices and type 2 diabetes and/or insulin resistance, and results were conflicting [12-15]. In addition, one study suggested that the association between DII and type 2 diabetes could be partly mediated by BMI. This result supported the hypothesis of an intermediate role of BMI in the association between DII and type 2 diabetes [12]. A previous study reported an interaction between DII and BMI in relation to type 2 diabetes with the association restricted to participants with BMI $\geq 25 \mathrm{~kg} / \mathrm{m}^{2}$ [15]. Taken together, the role of BMI is ambiguous.

Therefore, based on data from more than 70,000 E3N (Etude Epidémiologique auprès de femmes de l'Education Nationale) cohort study participants followed for 20 years, our aim is to prospectively assess the association between DII and type 2 diabetes and to evaluate the potential interaction and mediation of BMI in this relationship.

\section{Methods}

Study population and follow-up The E3N study is a large ongoing French prospective cohort of women, set up in 
France in 1990. The study protocol was reviewed and approved by the Commission Nationale de l'Informatique et des Libertés (CNIL); all participants gave written informed consent. The detailed protocol has been described elsewhere $[12,16]$. Briefly, we included 98,995 women born between 1925 and 1950, from the French national health insurance plan for teachers and co-workers, the Mutuelle Générale de l'Education Nationale. Women were enrolled in the cohort through a self-administered questionnaire, and were followed by self-administered questionnaires every 2 years on aspects including health conditions, lifestyle, diet, treatments and mental health status. Furthermore, for each cohort member, the health insurance plan provided data that included all outpatient reimbursements for health expenditure since 1 January 2004; these data included brand names, doses and dates of drug reimbursements. The average response rate to a followup questionnaire is $83 \%$, with a total loss to follow-up since 1990 below $3 \%$.

Population for analysis We initially included participants who had completed the dietary questionnaire sent in $1993(n=$ 74,522). Then we excluded all prevalent type 2 diabetes cases $(n=824)$, women with extreme energy intakes (i.e. below the 1 st and above the 99th percentiles of the energy intake over energy requirement distribution in the population) $(n=1491)$, and women who did not complete any follow-up questionnaire after the dietary questionnaire $(n=1216)$. The final study population included 70,991 women (see electronic supplementary material [ESM] Fig. 1). Follow-up commenced in 1993 (baseline for the present study) and ended in 2014 (latest date of type 2 diabetes case validation in the E3N cohort).

Dietary inflammatory index Diet was assessed using a validated 208-item semi-quantitative dietary questionnaire sent in 1993 [17]. Information on food and drink consumption was collected for eight consumption occasions throughout the day (breakfast, morning snack, aperitif before lunch, lunch, afternoon snack, pre-dinner aperitif, dinner and after dinner snack). Intakes of all food items were converted into intakes of energy and nutrients using food composition databases from the French Information Centre on Food Quality [18]. The validity and reproducibility of the dietary questionnaire have been previously described [17].

We calculated the DII using the adapted dietary inflammatory index (ADII) described by van Woudenbergh et al [12] as the starting point. This was derived from the literature-based score developed by Cavicchia et al [10] to reflect the inflammatory potential of an individual's diet. This DII has been proposed on the basis of nutritional rationale. First, the dietary inflammatory weights of dietary components are multiplied by the standardised energy-adjusted intake to reduce the between-person variation. Second, the intake of all components are standardised by subtracting the mean intake of the population from the individual intake and dividing the difference by the SD of the study to avoid the possibility that the variation in the ADII was solely driven by a few dietary components with a large range in intake. Finally, several components are excluded to avoid an overestimation of the inflammatory effects of ethanol, fat and energy. Thus, beer, wine, liquor and total fat were excluded. Energy was excluded because the inflammatory effect of energy was considered to be equivalent to the sum of the inflammatory effects of all energy-providing macronutrients. Dietary inflammatory weight for ethanol was assumed to be zero when the intake of ethanol was $>40 \mathrm{~g} /$ day because the intake of ethanol is not likely to be anti-inflammatory when an intake is $>40 \mathrm{~g} /$ day [12].

The DII weights proposed by Cavicchia et al [10] were updated with a more robust literature base, and 3 additional years of published data (2008-2010, inclusive), resulting in a slightly more than doubling of the total number of articles scored: 1943 were scored in the newer 2013 version compared with 929 in the older 2009 version [11]. Thus, we used these weights to calculate the DII. A total of 32 of the 40 possible dietary components were used for DII calculation (ESM Table 1) based on the food frequency questionnaire [17]. The DII is calculated in such a way that positive values indicate an anti-inflammatory diet and negative values correspond to a proinflammatory diet.

Ascertainment of type 2 diabetes Before 2004, all potential cases of type 2 diabetes were identified through follow-up questionnaires that included questions on the diagnosis of diabetes, diabetes-specific diet, diabetes drugs and hospitalisations for diabetes. All potential cases were then contacted and asked to answer a diabetes-specific questionnaire that included questions on the circumstances of diagnosis (year of diagnosis, symptoms, biological examinations, and fasting or random glucose concentration at diagnosis), diabetes therapy (prescription of diet or physical activity, list of all glucose-lowering drugs already used), and the most recent concentrations of fasting glucose and $\mathrm{HbA}_{1 \mathrm{c}}$. In order to be considered as validated for type 2 diabetes, an individual must have reported at least one of the following: (1) fasting plasma glucose $\geq 7.0 \mathrm{mmol} / 1$ or random glucose $\geq 11.1 \mathrm{mmol} / 1$ at diagnosis; (2) use of a glucose-lowering medication; (3) most recent values of fasting glucose concentrations $\geq 7.0 \mathrm{mmol} / 1$ or $\mathrm{HbA}_{1 \mathrm{c}}$ level $\geq 53 \mathrm{mmol} / \mathrm{mol}(7.0 \%)$ in the diabetes-specific questionnaire. After 2004, cases were identified through the drug reimbursement insurance database. The validation algorithm used in the $\mathrm{E} 3 \mathrm{~N}$ cohort to assess type 2 diabetes cases has been largely accepted and used in several previous publications (e.g. Fagherazzi et al [19]; Mancini et al $[20,21])$. All women who were reimbursed at least twice for any glucose-lowering medications during 1 year were considered to have validated diabetes [22]. 
Covariates All variables were obtained from the baseline questionnaire sent in 1993. BMI was calculated by dividing weight in kilograms by height in metres squared and was considered continuously in all models and in three categories for stratified analyses $\left(<20 \mathrm{~kg} / \mathrm{m}^{2}, 20-25 \mathrm{~kg} / \mathrm{m}^{2}\right.$, and $\geq 25 \mathrm{~kg} / \mathrm{m}^{2}$ ). The level of recreational physical activity (MET-h/week) was considered as a continuous variable. We considered three categories for smoking status (never, former and current), education level (undergraduate or less, graduate, and postgraduate or more), mentally tiring work (little or not, moderate and high) and personal history of hypercholesterolaemia (yes, no and unknown). Personal history of hypertension and family history of diabetes were in two categories (yes and no). Missing values were $<5 \%$ for all variables except for hypercholesterolaemia and therefore were imputed with the median and mode for quantitative and qualitative variables, respectively. For hypercholesterolaemia, an 'unknown' category was created to keep the same number of participants in the analyses.

Statistical analysis Baseline characteristics of the study population were reported as means (SD) for continuous variables and as numbers (percentages) for categorical variables.

We used Cox proportional hazards models with age as the timescale to estimate HR and 95\% CI. Participants were followed from age at baseline until age at diagnosis of type 2 diabetes, or age at death, or age at last follow-up, or age at the end of the follow-up period (2014), whichever occurred first. Schoenfeld residuals and log-minus-log plots were examined to confirm the proportional hazards assumption. No major violations were observed in any model.

DII was modelled in three different ways. For our main analysis, DII was included as a continuous variable, and we reported effect estimates for a 1-SD increase ( $z$ score) in DII. This approach assumes a linear association between DII and type 2 diabetes; to check the assumption, we then used multivariate restricted cubic spline with fives knots placed at the 5th, 27.5th, 50th, 72.5th and 95th percentiles of the DII distribution to provide a graphical presentation [23]. Splines allowed us to test whether there was a significant departure from a linear association. Finally, we categorised DII into quintiles and considered the first quintile group as the reference category in the Cox models.

Three models are presented: the first model was adjusted for age (timescale); the second was further adjusted for known type 2 diabetes risk factors and potential confounding factors: physical activity, smoking status, level of education, mentally tiring work, family history of diabetes, personal history of hypertension and hypercholesterolemia; the third model was further adjusted for BMI. The selection of confounders was done a priori, based upon the known risk factors of type 2 diabetes available in our dataset and associated with DII.
The interaction between DII and BMI on the risk of type 2 diabetes was estimated by including a multiplicative term between the two variables in the Cox model. As it was statistically significant, we examined the association of DII with type 2 diabetes stratified by BMI categories.

In addition to our main analysis described previously, and to better understand the complex relationship among DII, BMI and type 2 diabetes, we used counterfactual mediation models allowing for exposure-mediator interaction, according to which BMI represents both a mediator and an effect modifier of the relation between DII and type 2 diabetes [24]. This approach allows us to estimate several quantities for a change of DII from low to high: the controlled direct effect (CDE) is the average effect of an increase in DII, setting $\mathrm{BMI}=20 \mathrm{~kg} / \mathrm{m}^{2}$; the natural direct effect (NDE) represents the average effect of an increase in DII when BMI is fixed at the level it would take when DII is high; and the natural indirect effect (NIE) expresses how much the outcome would change on average when DII is controlled at a low level, but the mediator is changed from the level it would take if DII is high to the level it would take if DII is low. The total effect is the product of the NDE and NIE. In the absence of interaction, the CDE is equal to the NDE [24-26]. The proportion mediated is calculated using the following formula: $(\mathrm{HR} N \mathrm{NDE} \times(\mathrm{HR} \mathrm{NIE}-1)) /(\mathrm{HR} \mathrm{NDE} \times \mathrm{HR} \mathrm{NIE}-)$ [27]

Sensitivity analyses Several sensitivity analyses were performed. First, we repeated our main analysis when multiple imputations were applied for all covariates with missing data. Second, we excluded women with type 2 diabetes diagnosed in the first 5 years and the last 5 years of follow-up to examine whether reverse causation may partly explain our findings and whether inflammation through diet has a long term effect, respectively. Third, we further adjusted model 2 for adherence to the traditional Mediterranean diet score (range of scores, 0 to 9) [28], included as a continuous variable. Finally, we conducted similar analyses using three other DII definitions. The list of dietary components and the methods used for calculation of all DIIs are described in ESM Table 1.

Statistical analyses were performed using the Statistical Analysis System software, version 9.4 (SAS Institute, Cary, NC, USA).

\section{Results}

Baseline characteristics The inflammatory potential of the diet in our cohort, as measured by the DII, had a mean (SD) value of -0.06 (3.6). The range of the DII was from -13.42 to 24.8 ; the median, 25 th and 75 th percentiles were $-0.42,-2.5$, and 2.0 , respectively.

Table 1 presents the baseline characteristics of the study, overall and according to quintiles of DII. Overall, during a 
mean (SD) follow-up of 12.3 (5.4) years and 18.1 (2.0) years for participants with and without diabetes, respectively, a total of $3292(4.6 \%)$ validated incident type 2 diabetes cases were identified. The mean (SD) age of the population at baseline was 53 years (6.7). In general, with increasing DII quintile, mean age, BMI, and frequency of hypertension, hypercholesterolaemia and family history of diabetes decreased (Table 1).

DII and type 2 diabetes risk The association of DII with type 2 diabetes is presented in Table 2. In the first two models, DII was inversely associated with type 2 diabetes risk from the 2nd quintile group (HR 0.85 [95\% CI 0.77, 0.94]) up to the 5th quintile group (HR 0.77 [95\% CI 0.69, 0.85)], when compared with the 1st quintile group. Analysis using
1-SD increase in DII led to a similar result (model 2: HR 0.90 [95\% CI 0.87, 0.93]). Spline variables confirmed that there was no departure from a linear association $(p=0.797)$, and the graphical representation of the relation was consistent with a linear relation (Fig. 1). After inclusion of BMI (model 3), all associations were attenuated and became null (Table 2).

DII and type 2 diabetes risk stratified by BMI categories Table 3 shows the association between DII and type 2 diabetes stratified by categories of BMI. There was an interaction between DII and BMI on type 2 diabetes risk $\left(p_{\text {Interaction }}<0.0001\right)$. When stratified by BMI categories, the estimates were of similar magnitude, although, in women

Table 1 Baseline characteristics of the study population (E3N cohort data, $N=70,991$ )

\begin{tabular}{|c|c|c|c|c|c|c|}
\hline \multirow[b]{2}{*}{ Variable } & \multirow[b]{2}{*}{$\begin{array}{l}\text { Overall } \\
(N=70,991)\end{array}$} & \multicolumn{5}{|l|}{ Quintile of DII } \\
\hline & & $\begin{array}{l}\mathrm{Q} 1(<-3.00) \\
(N=14,199)\end{array}$ & $\begin{array}{l}\text { Q2 }(-3.00,-1.25) \\
(N=14,197)\end{array}$ & $\begin{array}{l}\text { Q3 }(-1.24,0.46) \\
(N=14,198)\end{array}$ & $\begin{array}{l}\text { Q4 }(0.47,2.69) \\
(N=14,198)\end{array}$ & $\begin{array}{l}\mathrm{Q} 5(\geq 2.70) \\
(N=14,199)\end{array}$ \\
\hline DII $(N=70,991)$ & $-0.06(3.60)$ & $-4.60(1.34)$ & $-2.07(0.50)$ & $-0.40(0.49)$ & $1.50(0.63)$ & $5.34(2.49)$ \\
\hline $\begin{array}{l}\text { T2D diagnosis at the end of } \\
\text { follow-up status }(\%)\end{array}$ & $3292(4.64)$ & $810(5.70)$ & $696(4.90)$ & $664(4.68)$ & $562(3.96)$ & $560(3.94)$ \\
\hline Age at baseline (years) & $52.88(6.67)$ & $53.22(6.50)$ & $53.25(6.65)$ & $53.18(6.74)$ & $52.71(6.69)$ & $52.04(6.68)$ \\
\hline \multicolumn{7}{|l|}{ Educational level (\%) } \\
\hline Undergraduate or less & $7879(11.09)$ & $1444(10.17)$ & $1526(10.75)$ & $1594(11.23)$ & $1608(11.33)$ & $1707(12.02)$ \\
\hline Graduate & $37,707(53.12)$ & $7427(52.31)$ & $7597(53.51)$ & 7705 (54.27) & $7629(53.73)$ & $7349(51.76)$ \\
\hline Postgraduate or more & $25,405(35.79)$ & $5328(37.52)$ & $5074(35.74)$ & $4899(34.50)$ & $4961(34.94)$ & $5143(36.22)$ \\
\hline Physical activity (MET-h/week) & $49.37(50.48)$ & $54.70(56.90)$ & $50.86(52.24)$ & $48.86(48.20)$ & $46.81(44.28)$ & $45.62(49.41)$ \\
\hline \multicolumn{7}{|l|}{ Smoking status (\%) } \\
\hline Current & $9581(13.50)$ & $2055(14.47)$ & $1841(12.97)$ & 1827 (12.87) & $1877(13.22)$ & $1981(13.95)$ \\
\hline Former & $23,257(32.76)$ & $5104(35.95)$ & 4840 (34.09) & $4610(32.47)$ & $4408(31.05)$ & $4295(30.25)$ \\
\hline Never & $38,153(53.74)$ & $7040(49.58)$ & $7516(52.94)$ & $7761(54.66)$ & $7913(55.73)$ & $7923(55.80)$ \\
\hline \multicolumn{7}{|l|}{ Mentally tiring work (\%) } \\
\hline Little or not mentally tiring & $15,606(21.98)$ & $3315(23.35)$ & $3128(22.03)$ & $3062(21.57)$ & $3045(21.45)$ & $3056(21.52)$ \\
\hline Mentally tiring & $43,809(61.71)$ & $8643(60.87)$ & $8854(62.37)$ & 8807 (62.03) & $8787(61.89)$ & $8718(61.40)$ \\
\hline Very mentally tiring & $11,576(16.31)$ & $2241(15.78)$ & $2215(15.60)$ & $2329(16.40)$ & $2366(16.66)$ & $2425(17.08)$ \\
\hline BMI $\left(\mathrm{kg} / \mathrm{m}^{2}\right)$ & $22.89(3.18)$ & $23.44(3.37)$ & $23.14(3.16)$ & $22.90(3.16)$ & $22.60(2.99)$ & $22.36(3.07)$ \\
\hline \multicolumn{7}{|l|}{ BMI categories $(\%)$} \\
\hline$<20\left(\mathrm{~kg} / \mathrm{m}^{2}\right)$ & $13,690(19.28)$ & $2247(15.83)$ & $2353(16.57)$ & $2686(18.92)$ & $2975(20.95)$ & $3429(24.15)$ \\
\hline $20\left(\mathrm{~kg} / \mathrm{m}^{2}\right) \geq \mathrm{BMI}<25\left(\mathrm{~kg} / \mathrm{m}^{2}\right)$ & $43,438(61.19)$ & 8419 (59.29) & $8756(61.68)$ & $8780(61.84)$ & $8871(62.48)$ & $8612(60.65)$ \\
\hline$\geq 25\left(\mathrm{~kg} / \mathrm{m}^{2}\right)$ & $13,863(19.53)$ & $3533(24.88)$ & $3088(21.75)$ & $2732(19.24)$ & $2352(16.57)$ & $2158(15.20)$ \\
\hline Hypertension (\%) & $36,590(51.54)$ & $7384(52.00)$ & $7421(52.27)$ & $7431(52.34)$ & $7198(50.70)$ & $7156(50.40)$ \\
\hline \multicolumn{7}{|l|}{ Hypercholesterolaemia (\%) } \\
\hline Yes & $5030(7.09)$ & $1158(8.16)$ & $1197(8.43)$ & $1065(7.50)$ & $914(6.44)$ & $696(4.90)$ \\
\hline Unknown & $7337(10.34)$ & $1617(11.38)$ & $1476(10.40)$ & $1445(10.18)$ & $1471(10.36)$ & $1328(9.35)$ \\
\hline Anti-inflammatory medications & $3836(5.40)$ & $825(5.81)$ & $773(5.44)$ & $784(5.52)$ & $696(4.90)$ & $758(5.34)$ \\
\hline Family history of diabetes (\%) & $7882(11.10)$ & $1712(12.06)$ & 1659 (11.69) & $1614(11.37)$ & $1480(10.42)$ & $1417(9.98)$ \\
\hline
\end{tabular}

Data are $N(\%)$ for categorical variables

Data are mean $(\mathrm{SD})$ for continuous variables

Q, quintile; T2D, type 2 diabetes 
Table 2 Risk of type 2 diabetes according to quintile groups of DII $(N=70,991)$

\begin{tabular}{|c|c|c|c|c|c|}
\hline & & & Model 1 & Model 2 & Model 3 \\
\hline & Participants without T2D $N(\%)$ & Participants with T2D $N(\%)$ & HR $(95 \% \mathrm{CI})$ & HR $(95 \% \mathrm{CI})$ & HR $(95 \%$ CI) \\
\hline For 1-SD increase & $N=67,699$ & $N=3292$ & $0.89(0.86,0.92)$ & $0.90(0.87,0.93)$ & $0.99(0.96,1.03)$ \\
\hline \multicolumn{6}{|l|}{ Quintile groups of DII } \\
\hline Q1 $(<-3.00)$ & $13,389(19.78)$ & $810(24.61)$ & Reference & Reference & Reference \\
\hline Q2 $(-3.00,-1.25)$ & $13,501(19.94)$ & $696(21.14)$ & $0.85(0.77,0.94)$ & $0.85(0.77,0.94)$ & $0.95(0.86,1.05)$ \\
\hline Q3 $(-1.24,0.46)$ & $13,534(19.99)$ & $664(20.17)$ & $0.81(0.73,0.90)$ & $0.82(0.74,0.91)$ & $0.95(0.86,1.06)$ \\
\hline Q4 $(0.47,2.69)$ & $13,636(20.14)$ & $562(17.07)$ & $0.70(0.63,0.78)$ & $0.73(0.65,0.81)$ & $0.92(0.82,1.03)$ \\
\hline Q5 ( $\geq 2.70)$ & $13,639(20.15)$ & $560(17.01)$ & $0.73(0.66,0.81)$ & $0.77(0.69,0.85)$ & $0.99(0.89,1.11)$ \\
\hline$p_{\text {Trend }}$ & & & $<0.001$ & $<0.001$ & 0.609 \\
\hline
\end{tabular}

Model 1: adjusted for age (as the timescale)

Model 2: further adjusted for family history of diabetes, smoking status, mentally tiring work, physical activity, educational level, hypercholesterolaemia and hypertension

Model 3: further adjusted for BMI

$\mathrm{T} 2 \mathrm{D}$, type 2 diabetes

with BMI $<20 \mathrm{~kg} / \mathrm{m}^{2}$, estimates were slightly higher, and the confidence intervals were wider.

Mediating role of BMI The results of the mediation analyses are presented in Fig. 2. First, we assumed a simple mediation model with no DII $\times$ BMI interaction on type 2 diabetes risk. Increasing DII was associated with lower type 2 diabetes risk, and an important proportion of the effect $(82 \%)$ was explained by a substantial indirect effect through BMI (NIE: HR 0.985 [95\% CI 0.984, 0.986]) (Fig. 2a). Then, in agreement with the interaction between DII and BMI on type 2 diabetes risk described above, we used mediation models allowing for exposure-mediator interaction, according to which BMI represents both a mediator and an effect modifier of the relation between DII and type 2 diabetes. Increasing DII was also associated with lower type 2 diabetes risk (HR 0.989 [95\% CI 0.980, 0.998] and HR 0.985 [95\% CI $0.984,0.986]$ for NDE and NIE, respectively), but the proportion explained by an indirect effect through BMI was lower (58\%) (Fig. 2b).

Sensitivity analyses Results were not substantially modified when multiple imputations were applied for all covariates with missing data (ESM Tables 2-3). Exclusion of participants with type 2 diabetes diagnosed in the first 5 years $(n=383)$ and in the last 5 years $(n=1009)$ of follow-up did not change the results (data not tabulated). Further adjustment for the Mediterranean dietary pattern did not change the results (ESM Fig. 2). There was a strong positive correlation between the four calculated DIIs (minimum $r=0.67 ; p<0.0001$ and maximum $r=0.96 ; p<0.0001)$. Analyses based on the three other DIIs led to the same patterns of association and interpretation (ESM Tables 4-9 and ESM Fig. 3).

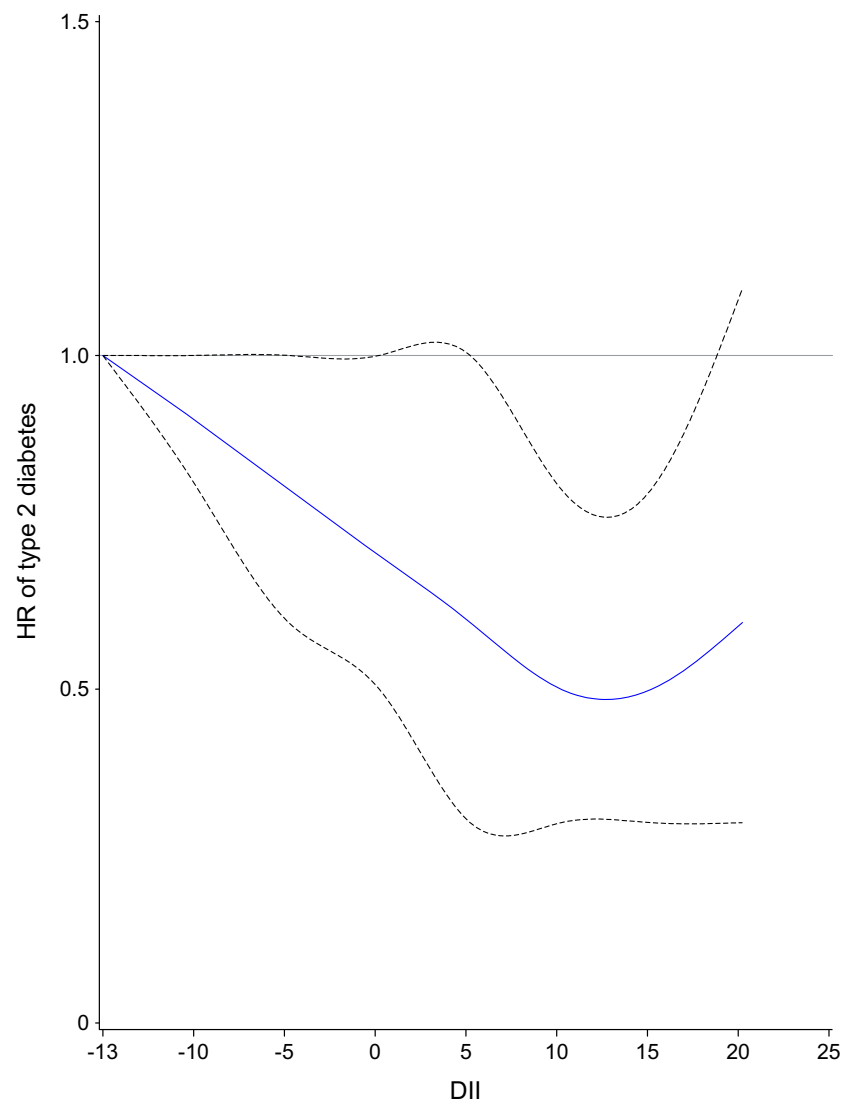

Fig. 1 Relationship between DII and hazard of type 2 diabetes fitted with restricted cubic splines (five knots placed at the 5th, 27.5th, 50th, 72.5th and 95 th percentiles). Risk estimates were adjusted for age, family history of diabetes, smoking status, mentally tiring work, physical activity, educational level, hypercholesterolaemia and hypertension. Reference value for HRs: minimum score (-13.42); the solid blue line represents the HR and the dashed lines the lower and upper $95 \% \mathrm{CI}$; $p_{\text {Overall }}<0.001$; $p_{\text {Nonlinear }}=0.797$ 
Table 3 Risk of type 2 diabetes by quintile of dietary inflammatory index stratified according to BMI $(N=70,991)$

\begin{tabular}{|c|c|c|c|c|c|}
\hline & & & Model 1 & Model 2 & $p_{\text {Interaction }}$ \\
\hline & $\begin{array}{l}\text { Participants without } \\
\text { T2D } N(\%)\end{array}$ & $\begin{array}{l}\text { Participants with } \\
\text { T2D } N(\%)\end{array}$ & $\mathrm{HR}(95 \% \mathrm{CI})$ & HR $(95 \% \mathrm{CI})$ & \\
\hline \multicolumn{6}{|c|}{$\mathrm{BMI}<20 \mathrm{~kg} / \mathrm{m}^{2}(N=10,256)$} \\
\hline For $1 \mathrm{SD}$ increase & $N=10,162$ & $N=94$ & $1.03(0.83,1.27)$ & $1.01(0.82,1.25)$ & $<0.0001$ \\
\hline Q1 $(<-3.00)$ & $1475(14.51)$ & $14(14.89)$ & Reference & Reference & \\
\hline Q2 $(-3.00,-1.25)$ & $1665(16.38)$ & $12(12.77)$ & $0.77(0.36,1.66)$ & $0.79(0.37,1.71)$ & \\
\hline Q3 $(-1.24,0.46)$ & $1963(19.32)$ & $23(24.47)$ & $1.23(0.63,2.40)$ & $1.18(0.61,2.30)$ & \\
\hline Q4 $(0.47,2.69)$ & $2285(22.49)$ & $21(22.34)$ & $1.00(0.51,1.96)$ & $0.97(0.49,1.92)$ & \\
\hline Q5 ( $\geq 2.70)$ & $2774(27.30)$ & $24(25.53)$ & $0.96(0.50,1.85)$ & $0.93(0.48,1.80)$ & \\
\hline \multicolumn{6}{|c|}{$20 \mathrm{~kg} / \mathrm{m}^{2} \geq \mathrm{BMI}<25 \mathrm{~kg} / \mathrm{m}^{2}(N=46,872)$} \\
\hline For $1 \mathrm{SD}$ increase & $N=45,488$ & $N=1384$ & $0.98(0.93,1.03)$ & $0.98(0.93,1.04)$ & \\
\hline Q1 $(<-3.00)$ & $8880(19.52)$ & $297(21.46)$ & Reference & Reference & \\
\hline Q2 $(-3.00,-1.25)$ & $9154(20.12)$ & $278(20.09)$ & $0.90(0.76,1.06)$ & $0.89(0.76,1.05)$ & \\
\hline Q3 $(-1.24,0.46)$ & $9200(20.23)$ & $280(20.23)$ & $0.90(0.77,1.06)$ & $0.90(0.77,1.06)$ & \\
\hline Q4 $(0.47,2.69)$ & $9273(20.39)$ & $267(19.29)$ & $0.88(0.75,1.04)$ & $0.89(0.75,1.05)$ & \\
\hline Q5 ( $\geq 2.70)$ & $8981(19.74)$ & $262(18.93)$ & $0.94(0.79,1.11)$ & $0.95(0.81,1.13)$ & \\
\hline \multicolumn{6}{|c|}{$\mathrm{BMI} \geq 25 \mathrm{~kg} / \mathrm{m}^{2}(N=13,863)$} \\
\hline For 1 SD increase & $N=12,049$ & $N=1814$ & $0.99(0.98,1.00)$ & $0.99(0.98,1.00)$ & \\
\hline Q1 $(<-3.00)$ & $3034(25.18)$ & $499(27.51)$ & Reference & Reference & \\
\hline $\mathrm{Q} 2(-3.00,-1.25)$ & $2682(22.26)$ & $406(22.38)$ & $0.91(0.80,1.04)$ & $0.91(0.80,1.04)$ & \\
\hline $\mathrm{Q} 3(-1.24,0.46)$ & $2371(19.68)$ & $361(19.90)$ & $0.93(0.81,1.06)$ & $0.93(0.81,1.07)$ & \\
\hline Q4 $(0.47,2.69)$ & $2078(17.25)$ & $274(15.10)$ & $0.82(0.71,0.95)$ & $0.84(0.73,0.98)$ & \\
\hline Q5 ( $\geq 2.70)$ & $1884(15.64)$ & $274(15.10)$ & $0.92(0.80,1.07)$ & $0.97(0.83,1.12)$ & \\
\hline
\end{tabular}

Model 1: adjusted for age (as the timescale)

Model 2: further adjusted for family history of diabetes, smoking status, mentally tiring work, physical activity, educational level, hypercholesterolaemia and hypertension

$p_{\text {Interaction }}$ was calculated using the multiplicative interaction term (DII $\left.\times \mathrm{BMI}\right)$

\section{Discussion}

In the E3N prospective cohort study of 70,991 women followed for 20 years, we showed an inverse linear association between baseline inflammatory properties of the diet and type 2 diabetes risk, independently of most known or potential risk factors or confounders. Women with higher DII (corresponding to an elevated anti-inflammatory potential of the diet) had a lower type 2 diabetes risk compared with those with lower DII (corresponding to a proinflammatory diet). The association was partly mediated by BMI and its interaction with DII.

Comparison with the literature The DII of our main analyses and those three versions considered in sensitivity analyses provided similar results although they are slightly different in their constructions. The range of DII (defined by Shivappa et al [11]) in our study (from -5.8 to +6.0 ) is similar to that reported in another French population $(-5.31$ to +6.26$)$ [29]. In addition, the range of the ADII (defined by van Woudenbergh) in our study (from -24.6 to +13.4 ) is also consistent with one reported in a Dutch population (from -12.0 to 15.7$)$ [12].

To our knowledge, our work is the first prospective study to show an inverse linear association of the proinflammatory potential of the diet with type 2 diabetes incidence. DII was developed to assess the overall inflammatory quality of the diet and it sums the individual pro-/anti-inflammatory effects of different dietary components as published in the literature [11]. Our findings are in line with some prospective studies that examined other diet quality indices such as the Mediterranean diet, and report a preventive role of a diet with an overall high quality with respect to type 2 diabetes incidence. For example, in large prospective studies, whole grain has been associated with both anti-inflammatory markers [30] and a decreased incidence of type 2 diabetes [30, 31]. In addition, a Mediterranean diet, recognised to be anti-inflammatory, has been associated with reduced type 2 diabetes risk [30-32]. In our study, the Mediterranean dietary pattern was moderately positively correlated with DII $(r=0.45)$ and further adjustment for it did not change the results, which 
Fig. 2 Mediation analyses without (a) and with (b) an interaction between DII and BMI on type 2 diabetes risk. ${ }^{\mathrm{a}} \mathrm{Cox}$ model adjusted for age, family history of diabetes, smoking status, mentally tiring work, physical activity, educational level, hypercholesterolaemia and hypertension. Estimates are mean HRs and are computed for changing the DII from 'low' (0.248 ) to 'high' (0.585); these values are the medians of DII in the two groups defined by clinically relevant cut-off (BMI $\geq$ $25 \mathrm{~kg} / \mathrm{m}^{2}$ and $\mathrm{BMI}<20 \mathrm{~kg} / \mathrm{m}^{2}$, respectively). Mean HRs below 1 indicate that increasing DII from low to high is associated with low type 2 diabetes risk. ${ }^{\mathrm{b}}$ The $95 \% \mathrm{CI}$ of these estimates was computed using the bootstrap method (1000 samples)

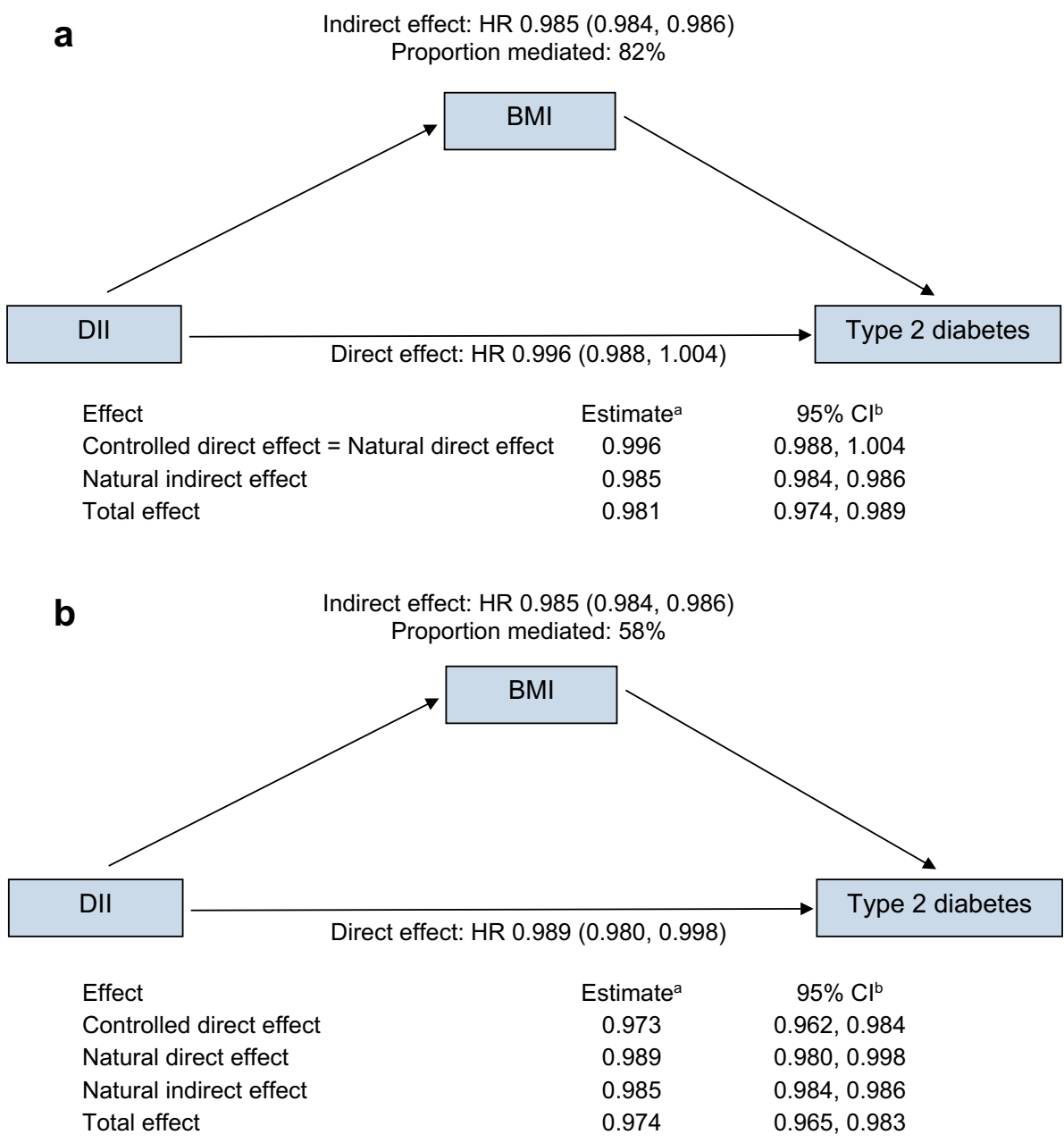

suggests that the potential anti-inflammatory effect of the diet is independent of the adoption of a Mediterranean diet. Our results are also consistent with some cross-sectional studies that reported an association between DII and type 2 diabetes and/or markers of glucose metabolism [12, 15, 33-36]. Van Woudenbergh et al reported that a proinflammatory diet was associated with higher fasting and postload glucose concentrations and higher insulin resistance, as quantified by the HOMA-IR index [12]. The authors also reported that lowgrade inflammation and BMI could mediate these associations, although modification by BMI was not investigated in that study. In addition, a recent study by Denova-Gutiérrez et al reported that a proinflammatory diet was associated with significantly higher odds of type 2 diabetes among adult Mexicans [15]. The authors also reported an effect modification of BMI with a proinflammatory diet that was not associated with type 2 diabetes in participants with BMI $<25 \mathrm{~kg} / \mathrm{m}^{2}$, but was associated in those with BMI $\geq 25 \mathrm{~kg} / \mathrm{m}^{2}$; however, there was no statistically significant interaction and the mediating role of BMI was not tested.

By contrast, our results disagree with the only prospective study that examined the association between DII and type 2 diabetes incidence; it included 592 participants, and it failed to show an association [12]. The relatively short follow-up time (mean 7.2 years) and the small sample size might explain the lack of association. In addition, other cross-sectional studies reported no association between DII and type 2 diabetes [14, 37]. These studies present some differences to the E3N study, including design, participants' sex (E3N included only women), number and types of dietary components used to calculate the DII (more than 30 dietary components were used in our E3N cohort in comparison with a mean of 27 in other studies) and availability of covariates for multivariable analyses.

Biological pathways As our analyses rely on observational data, our findings cannot be directly interpreted in terms of causality. Nevertheless, our findings are consistent with the hypothesis that diet plays a key role in the pathogenesis of type 2 diabetes. It is well known that certain foods and nutrients are capable of eliciting immunomodulatory effects and reducing inflammation, thereby improving beta cell function and insulin resistance [38]. Furthermore, the effect of antiinflammatory properties of the diet is partly mediated by BMI and interaction with BMI. The beneficial effect of anti- 
inflammatory diets could be partly mediated by their positive impact on BMI, one of the strongest risk factors in the development of type 2 diabetes. A previous study has shown that anti-inflammatory diets are negatively associated with glycaemic index score [34], and it was also shown that low glycaemic index or low glycaemic load diets resulted in weight loss in overweight and obese people, along with improvements in insulin sensitivity [34, 39-41]. The weight loss also caused a reduction in proinflammatory cytokines and increased anti-inflammatory cytokine production in adipose tissue $[42,43]$. The positive impact of anti-inflammatory properties of the diet could also be mediated by low-grade inflammation and other inflammatory parameters, especially those closely linked to adiposity $[44,45]$. In the E3N study, such parameters were not available.

Strengths and limitations Our study presents several strengths. First, the E3N study is a large prospective study with a long follow-up; exclusion of participants with type 2 diabetes diagnosed in the first 5 years of follow-up did not change the results, which suggests that reverse causation is unlikely to explain our findings. Second, we obtained stable results with similar conclusions when we used four different definitions of DII. Third, we were able to adjust our analyses for numerous type 2 diabetes risk factors as potential confounders. Fourth, the large number of participants, and the large number of participants with type 2 diabetes, ensured a high statistical power, however some stratified analyses where the number of participants with type 2 diabetes was more limited should be interpreted with caution. Finally, incident cases were identified from an algorithm based on an extensive medico-administrative database, which reduced the risk of missing or false-positive cases.

Our study also has limitations. First, the E3N cohort, like most cohort studies, is not representative of the French population. Therefore extrapolating results to the general population must be done carefully. Second, as the dietary consumption data used to calculate DII are self-reported, a certain degree of misclassification of exposure is possible and potential error measurement can never be ruled out. However, we used validated tools [17] in order to minimise the potential measurement error in the usual diet, and women with diets involving extreme energy intakes were excluded. In addition, as the study is prospective, any misclassification of exposure is likely to be non-differential and would result in an attenuation of associations. Third, because we have only dietary data from the 1993 wave, we have not been able to take into account changes in dietary habits over time. Fourth, although we controlled for confounding factors, we cannot exclude the possibility that unmeasured confounders may also influence our observations. However, it is improbable that such a bias would explain the consistently robust association observed between DII and type 2 diabetes. Fifth, our study population was exclusively women. This limitation should have been minor because no difference in biological mechanisms has been reported between men and women. Finally, mediation analyses under the counterfactual framework for causal inference are based on several assumptions that are difficult to verify, such as no unmeasured confounding or model misspecifications.

Conclusion In summary, our results suggest that a diet with a high anti-inflammatory potential is associated with a lower risk of type 2 diabetes and that adiposity, evaluated here with BMI, is one of the main mediators through which this may happen. These results help to improve our understanding of the mechanisms underlying the role of diet-related inflammation and type 2 diabetes. Furthermore, our findings support current diet-based approaches to prevent type 2 diabetes.

Acknowledgements The authors are indebted to all participants for their continued participation. They are also grateful to all members of the E3N study group.

Data availability The datasets generated during and/or analysed for the current study are not publicly available, but are available from the corresponding author on reasonable request.

Funding This work was supported by a grant for the E4N study project by the Agence Nationale de Recherche (ANR-10-COHO-0006 grant) and by a grant for the Nutriperso Project (IDEX Paris Saclay).

Duality of interest The authors declare that there is no duality of interest associated with this manuscript.

Contribution statement NL, FRM and GF conceived and designed the study. NL and GF performed the statistical analysis. NL and FRM drafted the original manuscript. All authors contributed to the interpretation of data discussed in the manuscript, revised the manuscript and approved its final version to be published. GF is the guarantor of this work and, as such, had full access to all the data in the study and takes responsibility for the integrity of the data and the accuracy of the data analysis.

\section{References}

1. International Diabetes Federation (2015) IDF Diabetes Atlas, 7th edn. Brussels, Belgium, IDF

2. Cho NH, Shaw JE, Karuranga S et al (2018) IDF Diabetes Atlas: Global estimates of diabetes prevalence for 2017 and projections for 2045. Diabetes Res Clin Pract 138:271-281. https://doi.org/10. 1016/j.diabres.2018.02.023

3. Hu FB, Meigs JB, Li TY, Rifai N, Manson JE (2004) Inflammatory markers and risk of developing type 2 diabetes in women. Diabetes 53(3):693-700. https://doi.org/10.2337/diabetes.53.3.693

4. Shoelson SE, Lee J, Goldfine AB (2006) Inflammation and insulin resistance. J Clin Invest 116(7):1793-1801. https://doi.org/10.1172/ JCI29069

5. Duncan BB, Schmidt MI, Pankow JS et al (2003) Low-grade systemic inflammation and the development of type 2 diabetes: the atherosclerosis risk in communities study. Diabetes 52(7):1799 1805. https://doi.org/10.2337/diabetes.52.7.1799 
6. Kang D-H, Rice M, Park N-J, Turner-Henson A, Downs C (2010) Stress and inflammation: a biobehavioral approach for nursing research. West J Nurs Res 32(6):730-760. https://doi.org/10.1177/ 0193945909356556

7. Smidowicz A, Regula J (2015) Effect of nutritional status and dietary patterns on human serum C-reactive protein and interleukin-6 concentrations. Adv Nutr 6(6):738-747. https://doi.org/10.3945/an. 115.009415

8. Minihane AM, Vinoy S, Russell WR et al (2015) Low-grade inflammation, diet composition and health: current research evidence and its translation. Br J Nutr 114(7):999-1012. https://doi.org/10. 1017/S0007114515002093

9. Nowlin SY, Hammer MJ, Melkus GD (2012) Diet, inflammation, and glycemic control in type 2 diabetes: an integrative review of the literature. J Nutr Metab 2012:1-21. https://doi.org/10.1155/2012/ 542698

10. Cavicchia PP, Steck SE, Hurley TG et al (2009) A new dietary inflammatory index predicts interval changes in serum highsensitivity C-reactive protein. J Nutr 139(12):2365-2372. https:// doi.org/10.3945/jn.109.114025

11. Shivappa N, Steck SE, Hurley TG, Hussey JR, Hébert JR (2014) Designing and developing a literature-derived, population-based dietary inflammatory index. Public Health Nutr 17(8):1689-1696. https://doi.org/10.1017/S1368980013002115

12. van Woudenbergh GJ, Theofylaktopoulou D, Kuijsten A et al (2013) Adapted dietary inflammatory index and its association with a summary score for low-grade inflammation and markers of glucose metabolism: the Cohort study on Diabetes and Atherosclerosis Maastricht (CODAM) and the Hoorn study. Am J Clin Nutr 98(6): 1533-1542. https://doi.org/10.3945/ajcn.112.056333

13. Vahid F, Shivappa N, Karamati M, Naeini AJ, Hebert JR, Davoodi SH (2017) Association between Dietary Inflammatory Index (DII) and risk of prediabetes: a case-control study. Appl Physiol Nutr Metab 42(4):399-404. https://doi.org/10.1139/apnm-2016-0395

14. Moslehi N, Ehsani B, Mirmiran P et al (2016) Inflammatory properties of diet and glucose-insulin homeostasis in a cohort of Iranian adults. Nutrients 8(11):735. https://doi.org/10.3390/nu8110735

15. Denova-Gutiérrez E, Muñoz-Aguirre P, Shivappa N et al (2018) Dietary inflammatory index and type 2 diabetes mellitus in adults: the Diabetes Mellitus Survey of Mexico City. Nutrients 10(4). https://doi.org/10.3390/nu10040385

16. Clavel-Chapelon F, for the E3N Study Group (2014) Cohort profile: the French E3N Cohort Study. Int J Epidemiol 44(3):801-809

17. van Liere MJ, Lucas F, Clavel F, Slimani N, Villeminot S (1997) Relative validity and reproducibility of a French dietary history questionnaire. Int J Epidemiol 26(Suppl 1):S128-S136. https:// doi.org/10.1093/ije/26.suppl_1.S128

18. de Securite Sanitaire (ANSES) AN (2013) Table de composition nutritionnelle des aliments Ciqual. www.ansespro.fr/ TableCIQUAL/. Accessed 12 Dec 2016

19. Fagherazzi G, Vilier A, Lajous M et al (2014) Wine consumption throughout life is inversely associated with type 2 diabetes risk, but only in overweight individuals: results from a large female French cohort study. Eur J Epidemiol 29(11):831-839. https://doi.org/10. 1007/s10654-014-9955-7

20. Mancini FR, Rajaobelina K, Praud D et al (2018) Nonlinear associations between dietary exposures to perfluorooctanoic acid (PFOA) or perfluorooctane sulfonate (PFOS) and type 2 diabetes risk in women: findings from the E3N cohort study. Int J Hyg Environ Health 221(7):1054-1060. https://doi.org/10.1016/j.ijheh. 2018.07.007

21. Mancini FR, Affret A, Dow C et al (2018) Dietary antioxidant capacity and risk of type 2 diabetes in the large prospective E3NEPIC cohort. Diabetologia 61(2):308-316. https://doi.org/10.1007/ s00125-017-4489-7
22. Mancini FR, Dow C, Affret A et al (2018) Micronutrient dietary patterns associated with type 2 diabetes mellitus among women of the E3N-EPIC (Etude Epidémiologique auprès de femmes de l'Education Nationale) cohort study. J Diabetes 10(8):665-674. https://doi.org/10.1111/1753-0407.12654

23. Harrell FE (2013) Regression modeling strategies: with applications to linear models, logistic regression, and survival analysis. Springer Science \& Business Media, New York

24. Valeri L, Vanderweele TJ (2013) Mediation analysis allowing for exposure-mediator interactions and causal interpretation: theoretical assumptions and implementation with SAS and SPSS macros. Psychol Methods 18(2):137-150. https://doi.org/10.1037/ a0031034

25. Ikram MA, Arfan Ikram M, VanderWeele TJ (2015) A proposed clinical and biological interpretation of mediated interaction. Eur $\mathrm{J}$ Epidemiol 30(10):1115-1118. https://doi.org/10.1007/s10654-0150087-5

26. Valeri L, VanderWeele TJ (2015) SAS macro for causal mediation analysis with survival data. Epidemiology 26(2):e23-e24. https:// doi.org/10.1097/EDE.0000000000000253

27. VanderWeele T (2015) Explanation in causal inference: methods for mediation and interaction. Oxford University Press, New York

28. Trichopoulou A, Naska A, DAFNE III Group (2003) European food availability databank based on household budget surveys: the Data Food Networking initiative. Eur J Pub Health 13(3 Suppl):24-28. https://doi.org/10.1093/eurpub/13.suppl_1.24

29. Neufcourt L, Assmann KE, Fezeu LK et al (2016) Prospective Association Between the Dietary Inflammatory Index and Cardiovascular Diseases in the SUpplémentation en VItamines et Minéraux AntioXydants (SU.VI.MAX) Cohort. J Am Heart Assoc 5(3): $\mathrm{e} 002735$

30. Ma Y, Hébert JR, Li W et al (2008) Association between dietary fiber and markers of systemic inflammation in the Women's Health Initiative Observational Study. Nutrition 24(10):941-949. https:// doi.org/10.1016/j.nut.2008.04.005

31. Liu S, Stampfer MJ, Hu FB et al (1999) Whole-grain consumption and risk of coronary heart disease: results from the Nurses' Health Study. Am J Clin Nutr 70(3):412-419. https://doi.org/10.1093/ajcn/ 70.3.412

32. InterAct Consortium, Romaguera D, Guevara M et al (2011) Mediterranean diet and type 2 diabetes risk in the European Prospective Investigation into Cancer and Nutrition (EPIC) study: the InterAct project. Diabetes Care 34(9):1913-1918. https://doi. org/10.2337/dc11-0891

33. Phillips CM, Shivappa N, Hébert JR, Perry IJ (2018) Dietary inflammatory index and biomarkers of lipoprotein metabolism, inflammation and glucose homeostasis in adults. Nutrients 10(8). https://doi.org/10.3390/nu10081033

34. Kim Y, Chen J, Wirth MD, Shivappa N, Hebert JR (2018) Lower dietary inflammatory index scores are associated with lower glycemic index scores among college students. Nutrients 10(2). https:// doi.org/10.3390/nu10020182

35. Farhangi MA, Najafi M (2018) Dietary inflammatory index: a potent association with cardiovascular risk factors among patients candidate for coronary artery bypass grafting (CABG) surgery. Nutr J 17(1). https://doi.org/10.1186/s12937-018-0325-2

36. Schulze MB, Hoffmann K, Manson JE et al (2005) Dietary pattern, inflammation, and incidence of type 2 diabetes in women. Am J Clin Nutr 82(3):675-684; quiz 714-5. https://doi.org/10.1093/ajcn/ 82.3.675

37. Alkerwi A‘a, Shivappa N, Crichton G, Hébert JR (2014) No significant independent relationships with cardiometabolic biomarkers were detected in the Observation of Cardiovascular Risk Factors in Luxembourg study population. Nutr Res 34(12):1058-1065. https://doi.org/10.1016/j.nutres.2014.07.017 
38. Pollack RM, Donath MY, Leroith D, Leibowitz G (2016) Antiinflammatory agents in the treatment of diabetes and its vascular complications. Diabetes Care 39(Supplement 2):S244-S252. https://doi.org/10.2337/dcS15-3015

39. Bouche C, Rizkalla SW, Luo J et al (2002) Five-week, lowglycemic index diet decreases total fat mass and improves plasma lipid profile in moderately overweight nondiabetic men. Diabetes Care 25(5):822-828. https://doi.org/10.2337/diacare.25.5.822

40. Neuhouser ML, Schwarz Y, Wang C et al (2011) A low-glycemic load diet reduces serum $\mathrm{C}$-reactive protein and modestly increases adiponectin in overweight and obese adults. J Nutr 142(2):369374. https://doi.org/10.3945/jn.111.149807

41. Joslowski G, Halim J, Goletzke J et al (2015) Dietary glycemic load, insulin load, and weight loss in obese, insulin resistant adolescents: RESIST study. Clin Nutr 34(1):89-94. https://doi.org/10. 1016/j.clnu.2014.01.015

42. Kern PA, Saghizadeh M, Ong JM, Bosch RJ, Deem R, Simsolo RB (1995) The expression of tumor necrosis factor in human adipose tissue. Regulation by obesity, weight loss, and relationship to lipoprotein lipase. J Clin Investig 95(5):2111-2119. https://doi. org/10.1172/JCI117899

43. Awad AB, Bradford PG (2009) Adipose tissue and inflammation. CRC Press Taylor \& Francis Group, Boca Raton. https://doi.org/10. 1201/9781420091311

44. Pahlavani M, Ramalho T, Koboziev I et al (2017) Adipose tissue inflammation in insulin resistance: review of mechanisms mediating anti-inflammatory effects of omega-3 polyunsaturated fatty acids. J Investig Med 65(7):1021-1027. https://doi.org/10.1136/ jim-2017-000535

45. Jayarathne S, Koboziev I, Park O-H, Oldewage-Theron W, Shen CL, Moustaid-Moussa N (2017) Anti-inflammatory and anti-obesity properties of food bioactive components : effects on adipose tissue. Prev Nutr Food Sci 22(4):251-262. https://doi.org/10.3746/pnf. 2017.22.4.251

Publisher's note Springer Nature remains neutral with regard to jurisdictional claims in published maps and institutional affiliations. 